\title{
HARDY-LITTLEWOOD MAXIMAL FUNCTION AND CLASS (Ap , Ap)
}

\author{
Santosh Ghimire \\ Department of Engineering Science and Humanities, Institute of Engineering, Pulchowk, Nepal
}

\begin{abstract}
In this article, we begin with class $\left(A_{p}, A_{p}\right)$ and Maximal function. We then establish the behavior of Maximal function on the functions of class $\left(\mathrm{A}_{\mathrm{p}}, \mathrm{A}_{\mathrm{p}}\right)$. Precisely, we show if the pair of weights $(u, w)$ is of class $\left(\mathrm{A}_{\mathrm{p}}, \mathrm{A}_{\mathrm{p}}\right)$ for some $1<\mathrm{p}<\infty$, then the Hardy Littlewood function M may not map $L^{p}(w)$ to $L^{p}(u)$. But in contrast if
\end{abstract} $(u, w) \in\left(\mathrm{A}_{\mathrm{p}}, \mathrm{A}_{\mathrm{p}}\right)$, the Maximal function M maps $L^{p}(w)$ to $L^{p, \infty}(u)$ with norm at most $C(n, p)[u, w]_{\left(A_{p}, A_{p}\right)}^{\frac{1}{p}}$.

Keywords: Maximal function, Hardy-Littlewood, Weight, Class (Ap, Ap)

\section{Introduction}

Some of the areas where the theory of weights is applied are vector-valued inequalities, extrapolation theory and estimates for certain class of non linear differential equation. Moreover, they are also widely used in the study of boundary value problems for Laplace's equation in Lipschitz domains. Muckenhoupt (1970) characterized positive functions $\mathrm{w}$ for which the Hardy-Littlewood maximal operator $\mathrm{M}$ maps $\mathrm{Lp}\left(\mathrm{R}^{\mathrm{n}}, \mathrm{w}(x) \mathrm{d} x\right)$ to itself. Muckenhoupt's characterization actually gave the better understanding of theory of weighted inequalities which then led to the introduction of $A_{p}$ class and then class $\left(A_{p}, A_{p}\right)$ and consequently the development of weighted inequalities. Weighted inequalities are used widely in harmonic analysis. Readers are suggested to refer $\mathrm{KC}$ and Ghimire(2016), Ghimire(2014 \&2016), and Parajuli and Ghimire(2015) for the theory of weighted inequalities, properties of weights and more.

Definition 1: The uncentered Hardy-Littlewood maximal operators on $\mathrm{R}^{\mathrm{n}}$ over balls $\mathrm{B}$ is defined as

$$
M(f)(x)=\sup _{x \in B} \operatorname{Avg}|f|=\sup _{x \in B} \frac{1}{|B|} \int_{B}|f(y)| d y .
$$

*Corresponding author: Santosh Ghimire

Department of Engineering Science and Humanities, Institute

of Engineering, Pulchowk, Nepal

Email: @santoshghimireioe.edu.np

(Received: 2016 Oct 02 Accepted: 2016 Nov 03)
Similarly the uncentered Hardy-Littlewood maximal operators on Rn over cubes Q is defined as

$$
M(f)(x)=\sup _{x \in Q} \operatorname{Avg}|f|=\sup _{x \in Q} \frac{1}{|Q|} \int_{Q}|f(y)| d y .
$$

In each of the definition above, the suprema are taken over all balls $\mathrm{B}$ and cubes $\mathrm{Q}$ containing the point $x$. H-L maximal functions are widely used in Harmonic Analysis. For the details about the H-L maximal operators, see Grafakos (1991) and Ba $\sim$ nelos and Moore (1991).

Definition 2: A locally integrable function on $\mathrm{R}^{\mathrm{n}}$ that takes values in the interval $(0, \infty)$ almost everywhere is called a weight. So by definition a weight function can be zero or infinity only on a set whose Lebesgue measure is zero.

We use the notation $w(E)=\int_{E} w(x) d x$ to denote the $\mathrm{w}$-measure of the set $\mathrm{E}$ and we reserve the notation $L^{p}\left(R^{n}, w\right)$ or $L^{p}(w)$ for the weighted $L^{p}$ spaces. We note that $\mathrm{w}(\mathrm{E})<\infty$ for all sets $\mathrm{E}$ contained in some ball since the weights are locally integrable functions.

Definition 3: A function $w(x) \geq 0$ is called an $A_{1}$ weight if there is a constant $\mathrm{C}_{1}>0$ such that $M(w)(x) \leq C_{1} w(x)$ 
where $\mathrm{M}(\mathrm{w})$ is uncentered Hardy-Littlewood Maximal function given by

$$
M(w)(x)=\sup _{x \in B} \frac{1}{|B|} \int_{B} w(t) d t .
$$

If $\mathrm{w}$ is an $\mathrm{A}_{1}$ weight, then the quantity (which is finite) given by

$$
[w]_{A_{1}}=\sup _{Q \text { cubes inR }}\left(\frac{1}{|Q|} \int_{Q}|w(t)| d t\right)\left\|w^{-1}\right\|_{L^{\infty}(Q)}
$$

is called the $A_{1}$ Muckenhoupt characteristic constant of $\mathrm{w}$ or simply $\mathrm{A}_{1}$ characteristic constant of $\mathrm{w}$.

Definition 4: Let $1<\mathrm{p}<\infty$. A weight $\mathrm{w}$ is said to be of class $A_{p}$ if $[w]_{A_{p}}$ is finite where $[w]_{A_{p}}$ is defined as

$[w]_{A_{p}}$

$=\sup _{Q \text { cubes in } \mathrm{R}^{\mathrm{n}}}\left(\frac{1}{|Q|} \int_{Q}|w(x)| d x\right)\left(\frac{1}{|Q|} \int_{Q}|w(x)|^{\frac{-1}{p-1}} d x\right)^{p-1}$.

Here $[w]_{A_{p}}$ is known as $\mathrm{A}_{\mathrm{p}}$ Muckenhoupt characteristic constant of $w$ or simply $A_{p}$ characteristic constant of $\mathrm{w}$. We remark that in the above definition of $A_{1}$ and $A_{p}$ one can also use set of all balls in $R^{n}$ instead of all cubes in $R^{n}$. Next we define class $\left(A_{p}, A_{p}\right)$.

Let $1<p<\infty$. A pair of weights $(u, w)$ is said to be of class $\left(\mathrm{A}_{\mathrm{p}}, \mathrm{A}_{\mathrm{p}}\right)$ if the quantity $[u, w]_{\left(\mathrm{A}_{\mathrm{p}}, \mathrm{A}_{\mathrm{p}}\right)}$ given by

$$
\begin{aligned}
& {[u, w]_{\left(\mathrm{A}_{\mathrm{p}}, \mathrm{A}_{\mathrm{p}}\right)}} \\
& =\sup _{Q \text { cubes in } \mathrm{R}^{\mathrm{n}}}\left(\frac{1}{|Q|} \int_{Q} u d x\right)\left(\frac{1}{|Q|} \int_{Q} w^{\frac{-1}{p-1}} d x\right)^{p-1}
\end{aligned}
$$

is finite and the quantity $[u, w]_{\left(\mathrm{A}_{\mathrm{p}}, \mathrm{A}_{\mathrm{p}}\right)}$ is called the $\left(A_{p}, A_{p}\right)$ characteristic constant of the pair $u$ and $w$. One can easily show that for any $L_{l o c}^{1}\left(R^{n}\right)$ function $\mathrm{f}$ with $0<\mathrm{f}<\infty$ a.e. and its Hardy-Littlewood Maximal function $M(f)$, the pair (f, $M(f)$ ) is of class $\left(A_{p}, A_{p}\right)$ for every $1<p<\infty$ with its characteristics constant independent of $\mathrm{f}$. We now state and prove our main results, named as Result 1 and Result 2. We begin with Result 1:

Result 1: If the pair of weight functions $(u, w)$ are of class $\left(A_{p}, A_{p}\right)$ for some $1<p<\infty$, then the Hardy-
Littlewood function $M$ may not map $L^{p}(w)$ to $L^{p}(u)$.

Proof: Let $g \geq 0$, integrable and nontrivial function such that its Hardy-Littlewood Maximal function $\mathrm{M}(\mathrm{g})$ does not belong to $L^{1}$. In addition to this, we take $g$ bounded so that $\mathrm{M}(\mathrm{g})$ is always finite. Then $(g, M g) \in\left(A_{1}, A_{1}\right) \subseteq\left(A_{p^{\prime}}, A_{p^{\prime}}\right)$ where $p^{\prime}=\frac{p}{p-1}$.

This now implies: $\quad\left((M g)^{\frac{-1}{p^{\prime}-1}},(g)^{\frac{-1}{p^{\prime}-1}}\right)=$ $\left((M g)^{1-p}, g^{1-p}\right) \in\left(A_{p}, A_{p}\right)$. Now we take $u=(M g)^{1-p}, w=g^{1-p}$. Then

$$
\begin{aligned}
& \int_{R^{n}}|M(f)(x)|^{p} u(x) d x= \\
& \int_{R^{n}}|M(g)(x)|^{p}|M(g)(x)|^{1-p} d x= \\
& \int_{R^{n}}|M(g)(x)| d x=\infty .
\end{aligned}
$$

On the other hand,

$$
\begin{gathered}
\int_{R^{n}}|f(x)|^{p} w(x) d x=\int_{R^{n}}|g|^{p}|g|^{1-p} d x \\
=\int_{R^{n}}|g(x)| d x<\infty .
\end{gathered}
$$

Thus the inequality,

$$
\begin{aligned}
\int_{R^{n}}|(M f)(x)|^{p} u(x) d x \\
\quad \leq c \int_{R^{n}}|f(x)|^{p} w(x) d x
\end{aligned}
$$

can't hold, in particular, for $\mathrm{f}=\mathrm{g}$. This proves that if $(u, w) \in\left(\mathrm{A}_{\mathrm{p}}, \mathrm{A}_{\mathrm{p}}\right)$, then the Hardy Littlewood function $\mathrm{M}$ may not map $L^{p}(w)$ to $L^{p}(u)$. This proves the Result 1 . Next we state our second result.

Result 2: If the pair of weights $(u, w)$ is of class $\left(A_{p}, A_{p}\right)$ for some $1<p<\infty$, then the Maximal function $M$ must map $L^{p}(w)$ to $L^{p, \infty}(u)$ with norm at most $C(n, p)[u, w]_{\left(A_{p}, A_{p}\right)}^{\frac{1}{p}}$.

In the course of proof, we will make the use of following problem whose proof is given:

Let $f \in L^{1}\left(R^{n}\right)$. Then for any $\alpha>0$, we show that there exist disjoint cubes $Q_{j}$ in $R^{n}$ such that the set $E_{\alpha}=\left\{x \in R^{n}: M_{c}(f)(x)>\alpha\right\}$ where $M_{c}(f)$ is the centered Maximal function, is contained in 
$\bigcup_{j} 3 Q_{j}$ and $\frac{\alpha}{4^{n}}=\frac{1}{\left|Q_{j}\right|} \int_{Q_{j}}|f(t)| d t \leq \frac{\alpha}{2^{n}}$.

Proof: Let $\alpha>0$ be given. We select all maximal dyadic cubes $Q_{j}(x)$ such that the average value of $\mathrm{f}$ over these dyadic cubes is bigger than $\alpha$. Let $x \in$ $E_{\alpha}$. Let us pick a cube $\mathrm{K}$ in $\mathrm{R}$ containing $x$ such that the average value of $|f|$ over $\mathrm{K}$ is bigger than $\alpha$ and we then find a dyadic cube $\mathrm{Q}$ such that $2^{-n}<|K| \leq$ $|Q|$ and also $\int_{K \cap Q}|f| d x>2^{-n} \alpha|K|$. One can easily find that $\mathrm{Q}$ is contained in some $Q_{j}\left(4^{-n} \alpha\right)$ and hence $\mathrm{K}$ is contained in $3 Q_{j}\left(4^{-n} \alpha\right)$. Then the collection of all dyadic cubes $Q_{j}=Q_{j}\left(4^{-n} \alpha\right)$ will satisfy the required inequality.

Proof of Result 2: Let $f \in L^{p}(w)$ and $Q^{\prime}$ be a cube in $R^{n}$. Then

$$
\begin{aligned}
& \left(\frac{1}{\left|Q^{\prime}\right|} \int_{Q^{\prime}}|f(x)| d x\right)^{p} u\left(Q^{\prime}\right) \\
& =\left(\frac{1}{\left|Q^{\prime}\right|} \int_{Q^{\prime}}|f| w^{\frac{1}{p}} W^{\frac{-1}{p}} d x\right)^{p} u\left(Q^{\prime}\right) \\
& \leq\left(\frac{1}{\left|Q^{\prime}\right|} \int_{Q^{\prime}}|f(x)|^{p} w d x\right)\left(\frac{1}{\left|Q^{\prime}\right|} \int_{Q^{\prime}} w^{\frac{p^{\prime}}{p}} d x\right)^{\frac{-p}{p^{\prime}}}\left(\frac{1}{\left|Q^{\prime}\right|} \int_{Q^{\prime}} u d x\right) \\
& \leq\left(\frac{1}{\left|Q^{\prime}\right|} \int_{Q^{\prime}}|f(x)|^{p} w d x\right)[u, w]_{\left(\mathrm{A}_{\mathrm{p}}, \mathrm{A}_{\mathrm{p}}\right)}
\end{aligned}
$$

Replacing f by $f \chi_{Q}$ with $Q \subseteq Q^{\prime}$,

$$
\begin{aligned}
& \left(\frac{1}{\left|Q^{\prime}\right|} \int_{Q^{\prime}}|f(x)| d x\right)^{p} u\left(Q^{\prime}\right) \\
& \quad \leq[u, w]_{\left(\mathrm{A}_{\mathrm{p}}, \mathrm{A}_{\mathrm{p}}\right)} \int_{Q}|f|^{p} w d x .
\end{aligned}
$$

Thus we have,

$$
u\left(Q^{\prime}\right) \leq[u, w]_{\left(\mathrm{A}_{\mathrm{p}}, \mathrm{A}_{\mathrm{p}}\right)}\left|Q^{\prime}\right| \frac{\int_{Q}|f|^{p} w d x}{\left(\int_{Q^{\prime}}|f| d x\right)^{p}}
$$

Using the problem as stated earlier, there exists disjoint cubes $Q_{j}$ such that $E_{\alpha}=\left\{x \in R^{n}: M_{c}(f)(x)>\alpha\right\} \subseteq \bigcup_{j=1}^{\infty} 3 Q_{j} \quad$ and $\frac{\alpha}{4^{n}}=\frac{1}{\left|Q_{j}\right|} \int_{Q_{j}}|f(t)| d t \leq \frac{\alpha}{2^{n}} . \quad$ Then $\quad u\left(E_{\alpha}\right) \leq$ $\sum_{j=1}^{\infty} u\left(3 Q_{j}\right)$. We take $Q^{\prime}=3 Q_{j}$ and $Q=Q_{j}$ in the above result, we have

$$
\begin{aligned}
& u\left(E_{\alpha}\right) \\
& \leq[u, w]_{\left(\mathrm{A}_{\mathrm{p}}, \mathrm{A}_{\mathrm{p}}\right)} \sum_{j=1}^{\infty} \frac{\left|3 Q_{j}\right|^{p}}{\left(\frac{1}{\left|Q_{j}\right|} \int_{Q_{j}}|f| d x\right)^{p}} \int_{Q_{j}}|f|^{p} w d x .
\end{aligned}
$$

$$
\begin{gathered}
\leq[u, w]_{\left(\mathrm{A}_{\mathrm{p}}, \mathrm{A}_{\mathrm{p}}\right)} \sum_{j=1}^{\infty} \frac{C(n, p)\left|Q_{j}\right|^{p}}{\left(\frac{1}{\left|Q_{j}\right|} \int_{Q_{j}}|f| d x\right)^{p}}\|f\|_{L^{p}\left(w_{j} ; Q_{j}\right)}^{p} \\
<[u, w]_{\left(\mathrm{A}_{\mathrm{p}}, \mathrm{A}_{\mathrm{p}}\right)} \frac{C^{\prime}(n, p)}{\alpha^{p}} \sum_{j=1}^{\infty}\|f\|_{L^{p}\left(w_{j} ; Q_{j}\right)}^{p} \\
<[u, w]_{\left(\mathrm{A}_{\mathrm{p}}, \mathrm{A}_{\mathrm{p}}\right)} \frac{C^{\prime}(n, p)}{\alpha^{p}}\|f\|_{L^{p}(w)}^{p}
\end{gathered}
$$

This gives

$$
\alpha u\left(E_{\alpha}\right)^{\frac{1}{p}} \leq[u, w]_{\left(\mathrm{A}_{\mathrm{p}}, \mathrm{A}_{\mathrm{p}}\right)}^{\frac{1}{p}} \frac{C^{\prime}(n, p)}{\alpha^{p}}\|f\|_{L^{p}(w)}^{p} .
$$

Now taking supremum over $\alpha$, we have

$$
\|M f\|_{L^{p, \infty}(u)} \leq[u, w]_{\left(\mathrm{A}_{\mathrm{p}}, \mathrm{A}_{\mathrm{p}}\right)}^{\frac{1}{p}} \frac{C^{\prime}(n, p)}{\alpha^{p}}\|f\|_{L^{p}(w)}^{p} .
$$

This proves that $\mathrm{M}$ maps $L^{p}(w)$ to $L^{p, \infty}(u)$ with norm at most $[u, w]_{\left(\mathrm{A}_{\mathrm{p}}, \mathrm{A}_{\mathrm{p}}\right)}^{\frac{1}{p}} C(n, p)$.

\section{References}

[1] Ba nelos, R \& Moore, C.N, 1991, Probabilistic Behavior of Harmonic Functions, Birkhauser Verlag.

[2] KC D.J., \& Ghimire, S., 2016 Relation Between BMO and $\mathrm{A}_{2}$ Function, Nepali Mathematical Sciences Report, Volume 34, No 1 and 2.

[3] Ghimire, S. 2014, Two Different Ways to Show a Function is an $\mathrm{A}_{1}$ Weight Function, Nepali Mathematical Sciences Report, Volume 33, No 1 and 2.

[4] Ghimire, S., 2014, Weighted Inequality, Journal of Institute of Engineering, Volume 10, No. 1, 2014.

[5] Ghimire,S., 2016, Some Properties of $A_{p}$ Weights Functions, Journal of Institute of Engineering, Volume 12, No. 1.

[6] Grafakos, L., 2009, Modern Fourier Analysis, Second Edition, Springer.

[7] Parajuli V. \& Ghimire, S. 2015, Product of $A_{p}$ Weight Functions, Journal of Institute of Engineering, Volume 11, No. 1. 